\title{
Distributed Monte Carlo Simulation of Light Transportation in Tissue
}

\author{
Andrew J. Page ${ }^{1}$, Shirley Coyle ${ }^{2}$, Thomas M. Keane ${ }^{1}$, Thomas J. Naughton ${ }^{1}$, \\ Charles Markham ${ }^{1}$, and Tomás Ward ${ }^{2}$ \\ ${ }^{1}$ Dept. of Computer Science \\ National University of Ireland \\ Maynooth, Co. Kildare, Ireland. \\ andrew.j.page@nuim.ie \\ ${ }^{2}$ Dept. of Electronic Engineering \\ National University of Ireland \\ Maynooth, Co. Kildare, Ireland. \\ tomas.ward@eeng.nuim.ie
}

\begin{abstract}
A distributed Monte Carlo simulation which models the propagation of light through tissue has been developed. It will allow for improved calibration of medical imaging devices for investigating tissue oxygenation in the white matter of the cerebral cortex. The application can distribute the simulation over an unbounded number of processors in parallel. We have found that this application is highly parallelisable resulting in up to $97 \%$ efficiency at 60 processors running on a homogeneous Java distributed system. A distributed system with 150 heterogeneous processors was used to simulate the paths of photons in a brain tissue model. We found that the source illumination footprint has an effect on the distribution of photons in the head and that lasers do produce a small beam in a highly scattering medium. This application will help researchers to improve the accuracy of their experiments.
\end{abstract}

\section{Introduction}

Near infrared spectroscopy has become a widely accepted method for investigating tissue oxygenation, in particular alterations in cerebral perfusion and oxygenation during functional activation of the brain. Light interaction with biological tissue is a complicated process due to the multiple layers of various cell types, each having different optical properties. In optical brain imaging studies light must first penetrate skin, skull and cerebrospinal fluid before reaching the grey and white matter of the cerebral cortex. Given the inhomogeneous nature of tissue it is important to develop adequate models to determine the nature of light propagation through tissue. This yields useful information for a number of optical diagnostic practises. A forward model of the propagation of light through the head is useful in solving the inverse problem in optical imaging studies, but also for determining irradiation doses in photodynamic therapy treatments.

Knowledge of the distribution of photon paths is the key to deciphering detected optical signals emerging from the surface of tissue. In near-infrared spectroscopic studies the photon path distribution is necessary for making quantitative measurements [7]. The highly scattering nature of biological tissue means that photons travel a considerably greater distance than the direct source-detector path. This distance, known as the differential pathlength, is needed to quantify absorption and scattering coefficients and consequently chromophore concentrations.

The spatial sensitivity profile of the photon path is important to ascertain firstly the volume of tissue interrogated and then which cells within that volume dominate the detected light signal. The relationship between penetration depth and source/detector spacing can be modelled which is an important factor for optode geometry and positioning. Applying this knowledge to determine light irradiance can help find light input and output powers, which is beneficial in choosing constituent components of an optical system, e.g. source power, and detector sensitivity.

A Java application has been developed to model the distribution of photon paths within tissue, utilising the Monte Carlo method. To generate useful results billions of photon paths must be simulated. On a single processor these simulations would take an inordinate amount of time, limiting the potential usefulness of the model. To address this limitation we have developed 
a distributed application which allows for, in theory, an unbounded number of processors to perform simulations in parallel. The application utilises the spare clock cycles of semi-idle PCs. In this paper we show that our distributed Monte Carlo application achieves 97\% efficiency when running on 60 homogeneous PCs.

The remainder of this paper is organised as follows. In Sect. 2 we discuss the Monte Carlo method and its applicability to this research. In Sect. 3 we describe the distributed computing element of the simulation. Some results are presented in Sect. 4. Finally in Sect. 5 we give our conclusions and future work.

\section{Monte Carlo method}

Light transport in tissue is analysed using radiative transport theory or the diffusion approximation [6]. The Monte Carlo method has been widely used as a numerical solution of the radiative transport theory equation [5] and it can be applied to an inhomogeneous medium of complex geometry once a realistic model of the tissue sample has been developed. Monte Carlo simulations have been used to study the effect of the superficial tissue thickness, which differs between adult and neonates, and also the effect of the cerebrospinal fluid, a layer of low scattering properties "sandwiched" between highly scattering tissue which has a significant effect on light propagation $[1,3]$.

Typical optical properties of the adult head are presented in Table 1, [1, 3]. The skin and scalp are highly scattering, as is the cerebral cortex. The CSF layer, which lies in between, has very low scattering properties and this confines the penetration of light to the shallow region of the grey matter, with few photons probing the white matter. Spatial sensitivity profiles of adult head models, calculated from the accumulated trajectories of photons, show that for source detector spacings of $20-60 \mathrm{~mm}$, the intensely sensitive region is confined to the grey matter. Increasing interoptode spacing does not allow absorption changes in the white matter to be calculated, but rather increases the volume of grey matter under investigation.

\section{Distributed Computing}

A general purpose distributed computation platform has been used to harness the spare computation resources of non-dedicated PCs [2] to simulate billions of photon paths through tissue. This would take an inordinate amount of time on a single processor.

The distributed Monte Carlo application consists of two classes. The DataManager, which resides on the

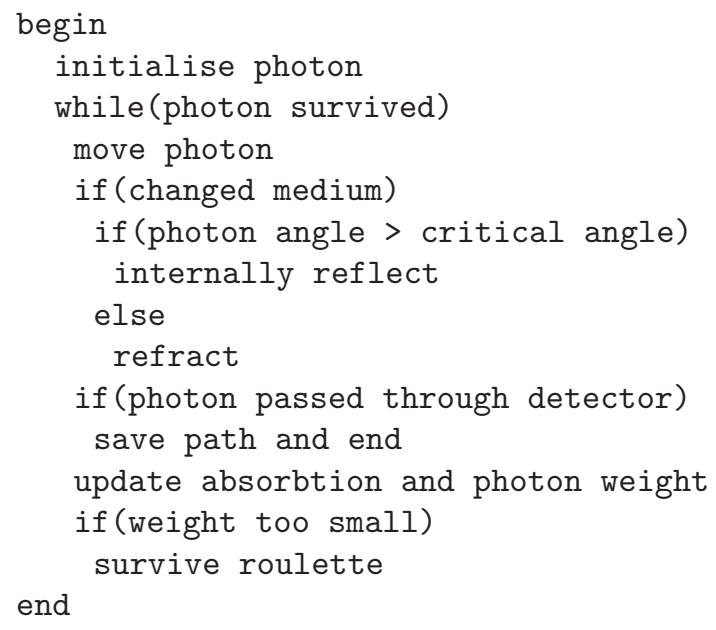

\section{Figure 1. Pseudocode of the Monte Carlo simluation}

server, assigns simulations to client PCs and processes the returned results. The Algorithm (see Fig. 1), which resides on the client $\mathrm{PCs}$, takes in parameters from the DataManager, performs Monte Carlo simulations and returns the results. The application provides a number of different features such as: allowing for different sources (delta, Gaussian, uniform), gated differential pathlengths, multiple user defined layers, refraction and internal reflection (classical physics or probabilistic methods), user defined granularity of results, and an unlimited number of simulations. In a real world experiment the pulse interferes with the paths taken by photons so the source and detector only operate between pulses. Thus the ability to gate the pathlengths allows for the simulation of this.

The speedup graph in Fig. 2 shows that as more processors are added to the distributed system, whilst processing the Monte Carlo simulation, a near linear speedup is achieved. Speedup is calculated as, $\frac{k P_{k}}{P_{1}}$ where $P_{1}$ is the time taken on 1 processor and $P_{k}$ is the time taken using $k$ processors. We used a homogeneous set of nondedicated PCs, Pentium IVs with 512MB RAM, for this speedup experiment. At 60 processors running the simulation the distributed system achieves over $97 \%$ efficiency. For further discussion on the efficiency of a system using heterogeneous processors see [4].

\section{Simulations}

For the experiments described in this section, we used a set of 150 heterogeneous clients. Table 2 details 


\begin{tabular}{|c|c|c|c|}
\hline Tissue Type & $\begin{array}{c}\text { Thickness } \\
(\mathrm{cm})\end{array}$ & $\begin{array}{c}\text { Transport Scattering } \\
\text { Coefficient } \mu_{s}^{\prime}\left(\mathrm{mm}^{-1}\right)\end{array}$ & $\begin{array}{c}\text { Absorption Coefficient } \\
\mu_{a}\left(\mathrm{~mm}^{-1}\right)\end{array}$ \\
\hline Scalp & $0.3-1$ & 1.9 & 0.018 \\
Skull & $0.5-1$ & 1.6 & 0.016 \\
CSF & 2 & 0.25 & 0.004 \\
Grey matter & 4 & 2.2 & 0.036 \\
White matter & - & 9.1 & 0.014 \\
\hline
\end{tabular}

Table 1. Thickness and Optical properties (NIR range) of Tissue in Adult Head

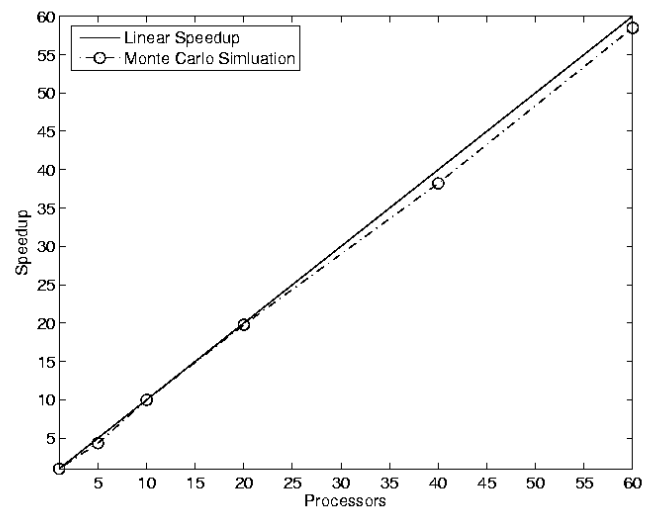

Figure 2. Speedup graph with varying numbers of homogeneous processors for the distributed Monte Carlo simulation

the processing rate of the processors in Mflop/s and the amount of memory available to the JVM. We had nondedicated usage of these processors, and the available processing and network resources varied stochastically over time. All the clients connected to a dedicated server running Linux (Fedora Core 4 ) on a $3 \mathrm{GHz} \mathrm{P} 4$ with 1 GB of RAM. In each simulation the paths taken by 1 billion photons were recorded, with each simulation taking approximately 2 hours on the distributed system detailed in Table 2 .

To verify the accuracy of the application, we mapped the paths of 1 billion photons through a homogeneous tissue (white matter). Only photon paths which reach the detector were counted. Fig. 3 shows the most common paths taken by the photons, after thresholding. The most common paths form a banana shape, as expected.

A model of the different layers of tissue in and around the brain has been created (as described in Ta-

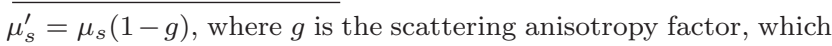
is the mean cosine of the scattering angle, therefore $g=-1$ means totally back-scattering; $g=0$ means isotropic scattering and $g=1$ means complete forward scattering.

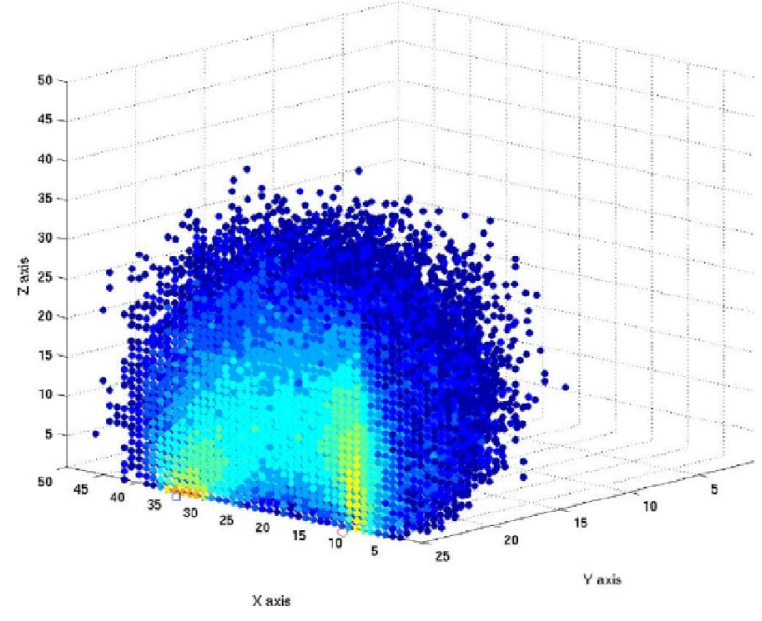

Figure 3. Simulation with a laser source and granularity of $50^{3}$ in homogeneous white matter tissue.

ble 1). Fig. 4 shows the results of this simulation. Most of the photons are reflected before they enter the CSF, however some do penetrate all the way into the white matter tissue, which is of most interest to researchers. The ability to model the statistics and distribution of the photon paths which reach the white matter tissue allows for more accurate calibration of the imaging experiments, which previously relied on trial and error.

\section{Conclusion \& Future Work}

Near infrared spectroscopy is a method for investigation of cerebral perfusion and oxygenation in the brain. It is important to develop adequate models to determine the nature of light propagation through tissue providing useful information for a number of optical diagnostic practises.

The Monte Carlo method has been widely used as a numerical solution of the radiative transport theory equation [5] and it can be applied to an inhomogeneous medium of complex geometry. 


\begin{tabular}{|r|r|r|l|l|}
\hline$\#$ & Mflop/s & RAM (MB) & O/S & Processor \\
\hline 91 & $28-31$ & 256 & Linux & P3 600MHz \\
50 & $190-229$ & 512 & Linux & P4 2.4GHz \\
4 & 15 & 192 & Linux & P2 266MHz \\
1 & 154 & 1024 & Windows XP & P4 Centrino $1.4 \mathrm{GHz}$ \\
1 & 25 & 512 & Linux & P3 $500 \mathrm{MHz}$ \\
1 & 37 & 256 & Linux & P3 $1 \mathrm{GHz}$ \\
1 & 72 & 256 & Linux & P4 $1.7 \mathrm{GHz}$ \\
1 & 91 & 1024 & FreeBSD & AMD $2400+\mathrm{XP}$ \\
\hline
\end{tabular}

Table 2. Distributed system resources

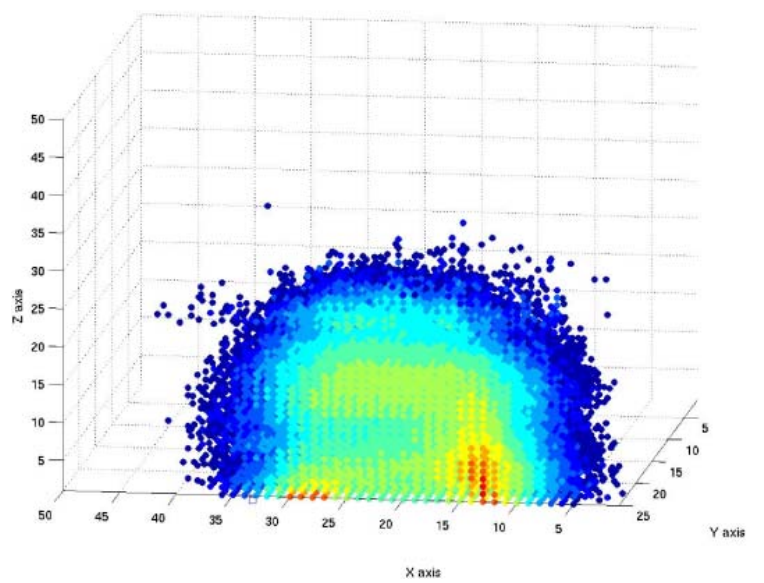

Figure 4. Simulated paths taken by photons with layers of brain tissue as defined in Table 1.

A Java distributed Monte Carlo simulation has been developed to model the distribution of photon paths in tissue. It has been used to test billions of paths with multiple layers of tissue. The application utilises the spare clock cycles of non-dedicated PCs achieving near linear speedup. The use of Java allowed for the use of an unbounded number of simulations, and for the rapid creation of a distributed application. Future work will concentrate on utilising the numerous features of the application to improve the calibration of the source and detector positions and sensitivities.

\section{References}

[1] Y. Fukui, Y. Ajichi, and E. Okada. Monte carlo prediction of near-infrared light propagation in realistic adult and neonatal head models. Applied Optics, 42(16):2881-2887, 2003.

[2] T. Keane, R. Allen, T. J. Naughton, J. McInerney, and J. Waldron. Distributed Java platform with programmable MIMD capabilities. In N. Guelfi, E. Aste- siano, and G. Reggio, editors, Scientific Engineering for Distributed Java Applications, volume 2604, pages 122-131. Springer Lecture Notes in Computer Science, February 2003.

[3] E. Okada and D. T. Delpy. Near-infrared light propagation in an adult head model i. modeling of low level scattering in the cerebrospinal fluid layer. Applied $\mathrm{Op}$ tics, 42(16):2906-2914, 2003.

[4] A. J. Page and T. J. Naughton. Framework for task scheduling in heterogeneous distributed computing using genetic algorithms. Artificial Intelligence Review, 24(3-4):415-429, Nov 2005.

[5] S. A. Prahl, M. Keijzer, S. L. Jacques, and A. J. Welch. A monte carlo model of light propagation in tissue. In G. J. Muller and D. H. Sliney, editors, Proceedings of Dosimetry of Laser Radiation in Medicine and Biology, volume 5, pages 102-111. SPIE, 1989.

[6] A. E. Profio. Light transport in tissue. Applied Optics, 28(12):2216-2222, 1989.

[7] J. S. Wyatt, M. Cope, D. T. Delpy, C. E. Richardson, A. D. Edwards, S. Wray, and E. O. R. Reynolds. Quantitation of cerebral blood volume in human infants by near-infrared spectroscopy. Journal of Applied Phyisology, 68:1086-1091, 1990. 